\title{
First-principles calculations of 002 structure factors for electron scattering in strained $\operatorname{In}_{x} \mathrm{Ga}_{1-x} \mathrm{As}$
}

\author{
A. Rosenauer* and M. Schowalter \\ Institut für Festkörperphysik, Universität Bremen, Otto-Hahn-Allee 1, 28359 Bremen, Germany \\ F. Glas \\ CNRS-Laboratoire de Photonique et de Nanostructures, Route de Nozay, 91460 Marcoussis, France \\ D. Lamoen \\ Departement Fysica, Universiteit Antwerpen, Groenenborgerlaan 171, B-2020 Antwerpen, Belgium
}

(Received 14 February 2005; published 12 August 2005)

\begin{abstract}
This work provides values of electron scattering 002 structure factors for $\operatorname{In}_{x} \mathrm{Ga}_{1-x}$ As as a function of the In concentration $x=0$ to 1 . These results allow accurate compositional analysis of pseudomorphically grown $\mathrm{In}_{x} \mathrm{Ga}_{1-x} \mathrm{As} / \mathrm{GaAs}$ layers by transmission electron microscopy methods relying on the chemical sensitivity of the (002) beam. The calculations go beyond the limits of the isolated atom approximation, because they take into account charge redistribution effects between atomic sites in the crystal, strain, and static atomic displacements. The computations were performed by the full potential linearized augmented plane-wave method using a generalized gradient approximation for the exchange and correlation part of the potential. The calculations of strained $\operatorname{In}_{x} \mathrm{Ga}_{1-x} \mathrm{As}$ correspond to the strain state in specimens with large, small, and intermediate thickness in the electron beam direction. Additionally, the effect of static atomic displacements is taken into account. All results are listed in a parameterized form. The calculated 002 structure factor vanishes at an In concentration of $16.4 \%$. This value is in a good agreement with previously reported experimental measurements. Hence, our results are a significant improvement with respect to the isolated atom approximation which is conventionally applied in transmission electron microscopy simulations, and which predicts a value of $22.5 \%$.
\end{abstract}

DOI: 10.1103/PhysRevB.72.085326

PACS number(s): 68.37.Lp, 71.15.Mb, 68.55.Nq

\section{INTRODUCTION}

In recent years, research on semiconductor nanostructures has increased because of the many possible applications in optoelectronic devices. In semiconductor laser structures, which is a main topic of research at present, the composition distribution of the active region strongly influences the performance of the device. However, the interrelation between growth parameters and composition distribution is not fully understood. A detailed investigation of growth processes requires accurate measurements of the composition distribution on an atomic scale.

Transmission electron microscopy (TEM) is a suitable tool to provide information on an atomic scale because of its high spatial resolution. Several methods have been developed to determine composition distributions in semiconductor nanostructures by analytical TEM or high resolution TEM. Among the first methods, energy dispersive spectroscopy (EDS) or electron energy loss spectroscopy ${ }^{1}$ (EELS) are applied. High resolution TEM methods include strain state analysis ${ }^{2,3}$ or techniques exploiting the chemical sensitivity of the (002) beam in sphalerite type materials. For these techniques, (002) dark field images ${ }^{4-6}$ or fringe images stemming from a two-beam interference of the transmitted beam and the (002) beam ${ }^{7}$ are used.

However, extraction of quantitative chemical information requires a comparison with measured calibration curves ${ }^{5,6}$ or with theoretical simulations. ${ }^{7}$ Such simulations are conventionally based on structure factors calculated from atomic scattering amplitudes, which can be found, e.g., in Refs. $8-10$. In these papers, atomic scattering amplitudes are computed for isolated atoms so that the redistribution of electrons due to bonding of atoms, which occurs in any specific crystal, cannot be taken into account in the structure factor calculation. As already shown by Zuo et al., ${ }^{11}$ charge redistribution effects can be taken into account by $a b$ initio density functional theory methods. The structure factors are also affected by local structural distortions which occur in most sphalerite type alloys because the atoms which share at least one of the two sublattices have different covalent radii. ${ }^{12}$ This effect can be taken into account by simulating large crystals and computing the corresponding static atomic displacements via the extended valence force field method. ${ }^{13}$

The interest of this work is to give values for the 002 structure factor as a function of $x$ in parameterized form, when the effects of charge redistribution between atomic sites in the crystal, strain, and static atomic displacements are taken into account. These data provide a useful basis for compositional analysis of strained and unstrained $\operatorname{In}_{x} \mathrm{Ga}_{1-x} \mathrm{As}$ layers by TEM using the chemically sensitive 002 beam. The aim of the paper is also to describe briefly the theoretical framework of the computations. In this paper we demonstrate how an $a b$ initio scheme using density functional theory and an empirical valence force field method which can be applied to large simulated crystals (containing typically one million atoms) can be combined to describe both charge redistribution effects and static atomic displacements.

In detail, we show that the redistribution of electrons due to bonding of atoms and the static atomic displacements sig- 
nificantly change the 002 structure factor of an $\operatorname{In}_{x} \mathrm{Ga}_{1-x} \mathrm{As}$ alloy. We show how the redistribution of electrons can be taken into account by introduction of modified atomic scattering amplitudes (MASAs). The MASAs are derived from $a b$ initio computations within the density functional theory formalism for different compositions of $\operatorname{In}_{x} \mathrm{Ga}_{1-x}$ As alloys. The dependence of the MASAs upon composition is conveniently fitted to third-order polynomials whose coefficients are given (Sec. II B). The advantage of using MASAs is the possibility to take into account static atomic displacements and Debye-Waller factors by appropriate correction factors. The correction factors for static displacements are computed using a valence force field model. Their dependence upon alloy composition is also fitted to third-order polynomials and coefficients are given (Sec. II C).

\section{COMPUTATION OF STRUCTURE FACTORS}

\section{A. Isolated atom approximation}

In TEM, amplitudes and phases of beams diffracted in a crystalline specimen can be computed by different approaches such as the Bloch wave method, ${ }^{14,15}$ the Multislice algorithm, ${ }^{16,17}$ or by solving the Darwin Howie-Whelan equations. ${ }^{18}$ These methods exploit Fourier coefficients of the crystal potential. In TEM simulations, it is common use to calculate the crystal potential in the isolated atom approximation, in which it is assembled from atomic potentials using tabulated atomic scattering amplitudes, such as those listed in parameterized form in Refs. 8-10. In the isolated atom approximation, the $h k l$ Fourier component of the potential of a binary (strained) sphalerite type crystal $\kappa$ (here GaAs or InAs) is given by (see, e.g., Ref. 19)

$$
\begin{aligned}
V_{\kappa}^{h k l}(\mathbf{a})= & \frac{h_{\mathrm{P}}^{2}}{2 \pi m_{0} e \Omega(\mathbf{a})} \sum_{\nu=1}^{2} \sum_{j=1}^{4} D_{\nu, \kappa}^{h k l}(\mathbf{a}) f_{\nu, \kappa}^{h k l}(\mathbf{a}) \\
& \times \exp \left[2 \pi i \mathbf{g}^{h k l}(\mathbf{a}) \cdot \mathbf{r}_{j, \nu}(\mathbf{a})\right],
\end{aligned}
$$

where $\mathbf{a}$ is a short hand notation for $\left\{\mathbf{a}_{i}\right\}, i=1,2,3$, and describes the lattice parameters of the unit cell along the [100], [010], and [001] direction (which may differ in a strained unit cell), $h_{\mathrm{P}}$ is Planck's constant, $e$ is the electron charge $(e=|e|), m_{0}$ is the rest mass of an electron, $\Omega(\mathbf{a})$ is the volume of the unit cell, $\nu$ indicates the sublattice (metal or non-metal), $f_{\nu, \kappa}^{h k l}(\mathbf{a})$ is the atomic scattering amplitude of an atom in the sublattice $\nu$ of a crystal $\kappa, D_{\nu, \kappa}^{h k l}(\mathbf{a})$ describes the damping of $f_{\nu, \kappa}^{h k l}(\mathbf{a})$ by thermal vibration, $j$ counts the four atoms in sublattice $\nu$ with positions $\mathbf{r}_{j, \nu}$ within the conventional (nonprimitive) unit cell of the crystal, and $\mathbf{g}^{h k l}(\mathbf{a})$ is the reciprocal lattice vector. For the cubic and orthorhombic crystal structures considered in this work (pertaining respectively to bulk and epitaxial samples), it is given by

$$
\mathbf{g}^{h k l}(\mathbf{a})=\left(\frac{h}{a_{[100]}}, \frac{k}{a_{[010]}}, \frac{l}{a_{[001]}}\right),
$$

where the factor " $2 \pi$ " has been excluded, in accordance with the conventions used in the International Tables for Crystallography. ${ }^{20}$ The damping factor $D_{\nu, \kappa}^{h k l}(\mathbf{a})$ depends on the temperature dependent Debye-Waller factor $B_{\nu, \kappa}(T)$ according to

$$
D_{\nu, \kappa}^{h k l}(\mathbf{a})=\exp \left\{-\frac{1}{4} B_{\nu, \kappa}(T)\left[\mathbf{g}^{h k l}(\mathbf{a})\right]^{2}\right\} .
$$

In the isolated atom approximation, the atomic scattering amplitudes can be derived from

$$
f_{\nu, \kappa}^{h k l}(\mathbf{a})=\left(1+\frac{e U}{m_{0} c^{2}}\right) \sum_{i=1}^{4} a_{\nu, \kappa, i} \exp \left(-\frac{1}{4} b_{\nu, \kappa, i}\left[\mathbf{g}^{h k l}(\mathbf{a})\right]^{2}\right),
$$

where the coefficients $a_{\nu, \kappa, i}$ and $b_{\nu, \kappa, i}$ are listed in Ref. 8. Note that in our definition the relativistic correction (1 $\left.+e U / m_{0} c^{2}\right)$ necessary to describe the interaction of the highenergy electron with the crystal potential in the TEM theory is included in the atomic scattering amplitudes.

Equation (1) is connected with the structure factor for electron scattering (in units of meters) by

$$
F_{\kappa}^{h k l}(\mathbf{a})=\frac{2 \pi m_{0} e \Omega(\mathbf{a})}{h_{\mathrm{P}}^{2}} V_{\kappa}^{h k l}(\mathbf{a}) .
$$

In the ternary semiconductor $\operatorname{In}_{x} \mathrm{Ga}_{1-x}$ As the metal sublattice is occupied by a random distribution of In and $\mathrm{Ga}$ atoms. The atomic scattering amplitude of atoms in the metal sublattice can thus be approximated by a linear combination of the scattering amplitudes of In and Ga. As a consequence, the crystal potential with which the fast electron interacts can in good approximation be described by (cf. virtual crystal approximation for randomly disordered alloys)

$$
V_{\mathrm{In}_{x} \mathrm{Ga}_{1-x} \mathrm{As}}^{h k l}(\mathbf{a})=x V_{\mathrm{InAs}}^{h k l}(\mathbf{a})+(1-x) V_{\mathrm{GaAs}}^{h k l}(\mathbf{a}) \text {. }
$$

Now we consider the case of a thin $\operatorname{In}_{x} \mathrm{Ga}_{1-x} \mathrm{As}$ layer buried in GaAs and derive an expression for the vector a describing the lattice parameters of the unit cell along the different $\langle 100\rangle$ directions. Chemical TEM analysis of $\mathrm{In}_{x} \mathrm{Ga}_{1-x} \mathrm{As}$ heterostructures with a chemically sensitive $\{002\}$ beam is usually carried out with an orientation of the specimen where the incident electron beam is close to a $\langle 100\rangle$ direction of the crystal. Another choice would be an orientation close to a $\langle 110\rangle$ direction. According to dynamic diffraction theory (see, e.g., Refs. 19, 21, and 22), the strong $\{111\}$ beams would then contribute to the weak (002) beam by multiple scattering, which complicates the measurement of the chemical composition. Therefore, we restrict our considerations to an orientation close to a $\langle 100\rangle$ direction which is better suited for compositional analysis. Without loss of generality we assume an [001] growth direction of the heterostructure, and an electron beam direction close to [010].

An $\mathrm{In}_{x} \mathrm{Ga}_{1-x}$ As layer pseudomorphically grown on GaAs is biaxially stressed. Using the definitions of directions made in the preceding, this leads to a tetragonal distortion along the [001] direction. For an investigation of the specimen in the TEM, the specimen thickness along the [010] direction has to be sufficiently small, typically between 5 and $50 \mathrm{~nm}$. In such a thin TEM specimen, the strained $\operatorname{In}_{x} \mathrm{Ga}_{1-x}$ As layer can expand close to the (010) surfaces, which leads to a reduction of the tetragonal distortion. In case of an "infinitely" thin specimen, the biaxial stress is reduced to uniaxial stress applied along the [100] direction. For a realistic speci- 
men thickness, the strain state lies in between the uniaxial and biaxial states. Applying elasticity theory, the lattice parameter $\mathbf{a}$ of a strained $\operatorname{In}_{x} \mathrm{Ga}_{1-x}$ As layer is given by

$$
\mathbf{a}(x, s)=a_{\text {bulk }}(x)[\mathbf{1}+\epsilon(x, s)],
$$

where $a_{\text {bulk }}(x)$ is the bulk lattice parameter of $\operatorname{In}_{x} \mathrm{Ga}_{1-x} \mathrm{As}$. In analogy to the definition of $\mathbf{a}, \epsilon(x, s)$ is a short hand notation for the dilatational strain components $\left\{\boldsymbol{\epsilon}_{i}\right\}, i=1,2,3$ along the [100], [010], and [001] directions as a function of $x$ and $s$, given by

$$
\begin{gathered}
\epsilon_{[100]}(x, s)=f(x), \\
\epsilon_{[010]}(x, s)=f(x) \frac{s\left[c_{11}(x)+c_{12}(x)\right]-c_{12}(x)}{c_{11}(x)+c_{12}(x)(1-s)}, \\
\epsilon_{[001]}(x, s)=-f(x) \frac{(1+s) c_{12}(x)}{c_{11}(x)+c_{12}(x)(1-s)},
\end{gathered}
$$

where the $c_{i j}(x)$ are the elastic constants of $\operatorname{In}_{x} \mathrm{Ga}_{1-x}$ As which can be approximated by

$$
c_{i j}(x)=x c_{i j}(1)+(1-x) c_{i j}(0),
$$

where the $c_{i j}(1)$ and $c_{i j}(0)$ are the elastic constants of InAs and GaAs, respectively. The misfit $f(x)$ is given by

$$
f(x)=\frac{a_{\text {bulk }}(0)-a_{\text {bulk }}(x)}{a_{\text {bulk }}(x)} .
$$

In Eq. (8), $s$ is a parameter that describes the specimen thickness in [010] direction. The value $s=1$ corresponds to the limit of an infinitely thick specimen, and $s=0$ describes an infinitely thin specimen. In the following, we denote a specimen thickness for which $s=0.5$ as "intermediate" thickness. Note however, that the true thickness which corresponds to $s=0.5$ depends on the profile of the In-concentration $x$ within the layer, and on the height of the layer in [001] growth direction. For a given layer height and a given Inconcentration profile, the specimen thickness corresponding to $s=0.5$ may be found by finite-element calculations.

\section{B. Density functional theory methods}

\section{Theoretical considerations}

The isolated atom approximation does not take into account redistribution of electrons in chemical bonds. Since this effect mainly alters the charge distribution between the atoms, the Fourier components of the crystal potential with small spatial frequencies are affected most. Therefore, it can be expected that the isolated atom approximation leads to significant errors for the calculation of the 002 structure factor. Density functional theory (DFT) methods can be used to compute the crystal potential taking into account redistribution of electrons in bonds. ${ }^{23}$ Our calculations were performed with the "WIEN2k" program package. ${ }^{24}$ Crystal potentials computed by DFT methods correspond to the ground state of a semiconductor, i.e., to a temperature $T=0$. Therefore, a direct use of crystal potential does not allow to account for the effect of thermal vibrations of atoms. In the isolated atom approximation in Eq. (1), this effect is included by the Debye-Waller factors. In order to use element (and crystal) dependent Debye Waller factors also for structure factors resulting from the DFT calculation, we applied the following procedure: We used the "lapw3" program of the WIEN2k package to calculate $\mathrm{x}$-ray scattering structure factors. In the WIEN2k program, the crystal is subdivided into spheres around the atoms and the interstitial region in between the spheres. For our calculations we chose the radius $R_{\mathrm{MT}}$ of the spheres ("muffin-tin radius") $10 \%$ smaller than half the nextneighbor distance. The lapw3 program lists separate contributions to an x-ray scattering structure factor stemming from spheres of nonequivalent atoms and the interstitial region. For a GaAs crystal, we, e.g., obtain the value $X_{\mathrm{Ga}}^{h k l}$ that corresponds to the contribution from all the spheres around $\mathrm{Ga}$ atoms, the value $X_{\mathrm{As}}^{h k l}$ from the spheres around the As atoms and the value $X_{\text {Out }}^{\text {hkl }}$ from the interstitial region outside the spheres. The total $\mathrm{x}$-ray scattering structure factor for a reflection $h k l$ is then given by

$$
\begin{aligned}
X_{\mathrm{GaAs}}^{h k l}(\mathbf{a}) & =X_{\mathrm{Ga}, \mathrm{GaAs}}^{h k l}(\mathbf{a})+X_{\mathrm{As}, \mathrm{GaAs}}^{h k l}(\mathbf{a})+X_{\mathrm{Out}, \mathrm{GaAs}}^{h k l}(\mathbf{a}) \\
& =\sum_{\nu=1}^{3} X_{\nu, G a A s}^{h k l}(\mathbf{a}) .
\end{aligned}
$$

Note that $X_{\mathrm{GaAs}}^{h k l}(\mathbf{a})$ is proportional to the $(h k l)$ component of the Fourier transform of the electron density and therefore is only valid for nonresonant $\mathrm{x}$-ray scattering. Using the MottBethe formula, ${ }^{25}$ the relativistically corrected potential for electrons in a binary semiconductor crystal $\kappa$, when they are accelerated through a potential $U$, can be expressed as

$$
\begin{aligned}
V_{\kappa}^{h k l}(\mathbf{a})= & \frac{e\left(1+\frac{e U}{m_{0} c^{2}}\right)}{4 \pi^{2} \epsilon_{0}\left[\mathbf{g}^{h k l}(\mathbf{a})\right]^{2} \Omega(\mathbf{a})} \\
& \times\left\{\sum_{\nu=1}^{2} \sum_{j=1}^{4} D_{\nu, \kappa}^{h k l}(\mathbf{a}) Z_{\nu, \kappa} \exp [2 \pi i\right. \\
& \left.\times \mathbf{g}^{h k l}(\mathbf{a}) \cdot \mathbf{r}_{j, \nu}(\mathbf{a})\right] \\
& \left.-\sum_{\nu=1}^{3} D_{\nu, \kappa}^{h k l}(\mathbf{a}) X_{\nu, \kappa}^{h k l}(\mathbf{a})\right\},
\end{aligned}
$$

where $Z_{\nu, \kappa}$ is the nuclear charge, $c$ is the speed of light in vacuum, and $\epsilon_{0}$ is the dielectric constant in vacuum. In Eq. (12), the same Debye-Waller factors are applied to the nuclear charges and the electron charge represented by $X_{\nu, \kappa}^{h k l}(\mathbf{a})$ (adiabatic approximation). Note that the second sum over $\nu$ expands from 1 to 3 , corresponding to the spheres around the metal atoms $(\nu=1)$, those around the nonmetal atoms $(\nu=2)$, and the region outside of the spheres $(\nu=3)$. Since the contribution of the region outside the spheres to the $\mathrm{x}$-ray scattering structure factor usually is much smaller than the contribution from the spheres $\left[X_{3, \kappa}^{h k l}(\mathbf{a}) \ll X_{(1,2), \kappa}^{h k l}(\mathbf{a})\right]$, the choice of $D_{3, \kappa}^{h k l}(\mathbf{a})$ is not critical. We use $D_{3, \kappa}^{h k l}(\mathbf{a})=\frac{1}{2}\left[D_{1, \kappa}^{h k l}(\mathbf{a})\right.$ $\left.+D_{2, \kappa}^{h k l}(\mathbf{a})\right]$. 
In the next step, Eq. (12) is rewritten in a form similar to Eq. (1), which allows the identification of "modified atomic scattering amplitudes"

$$
\begin{aligned}
V_{\kappa}^{h k l}(\mathbf{a})= & \frac{e\left(1+\frac{e U}{m_{0} c^{2}}\right)}{4 \pi^{2} \epsilon_{0}\left[\mathbf{g}^{h k l}(\mathbf{a})\right]^{2} \Omega(\mathbf{a})} \sum_{\nu=1}^{2} \sum_{j=1}^{4} D_{\nu, \kappa}^{h k l}(\mathbf{a})\left[Z_{\nu, \kappa}-\frac{X_{\nu, \kappa}^{h k l}(\mathbf{a})}{\tau_{\nu}}\right. \\
& \left.-\frac{X_{3, \kappa}^{h k l}(\mathbf{a})}{2 \tau_{\nu}}\right] \exp \left[2 \pi i \mathbf{g}^{h k l}(\mathbf{a}) \cdot \mathbf{r}_{j, \nu}(\mathbf{a})\right],
\end{aligned}
$$

with

$$
\tau_{\nu}=\sum_{j=1}^{4} \exp \left[2 \pi i \mathbf{g}^{h k l}(\mathbf{a}) \cdot \mathbf{r}_{j, \nu}(\mathbf{a})\right] .
$$

In Eq. (13) we added the X-ray structure factor $X_{3, \kappa}^{h k l}(\mathbf{a})$ of the outer regions of the spheres equally to the $\mathrm{X}$-ray structure factor of the metal and nonmetal atoms. A comparison of Eq. (13) with Eq. (1) leads to the definition of MASAs $f_{\nu, \kappa}^{\prime h k l}$ that replace the atomic scattering amplitudes $f_{\nu, \kappa}^{h k l}$ in Eq. (1) and which take into account the redistribution of electrons in bonds

$$
f_{\nu, \kappa}^{\prime h k l}(\mathbf{a})=\frac{e^{2} m_{0}\left(1+\frac{e U}{m_{0} c^{2}}\right)}{2 \pi h_{\mathrm{P}}^{2} \epsilon_{0}\left[\mathbf{g}^{h k l}(\mathbf{a})\right]^{2}}\left[Z_{\nu, \kappa}-\frac{X_{\nu, \kappa}^{h k l}(\mathbf{a})}{\tau_{\nu}}-\frac{X_{3, \kappa}^{h k l}(\mathbf{a})}{2 \tau_{\nu}}\right] .
$$

Note that MASAs are calculated for a special binary crystal, so that, e.g., the MASA for As atoms in GaAs $\left(f_{\mathrm{As}, \mathrm{GaAs}}^{\prime h k l}\right)$ is different from the MASA of As in InAs $\left(f_{\mathrm{As}, \mathrm{InAs}}^{\prime \prime h k l}\right)$. The advantage of using the MASAs is the possibility to include Debye-Waller temperature factors, which is necessary if the computed structure factors are applied for compositional analysis.

\section{Results}

For the computation of the 002 structure factors for electron diffraction in ternary $\operatorname{In}_{x} \mathrm{Ga}_{1-x}$ As we applied the procedure described in the following. For a certain Inconcentration $x$ and strain factor $s$ [Eq. (8)] we obtained the lattice parameter $\mathbf{a}(x, s)$ of the strained $\mathrm{In}_{x} \mathrm{Ga}_{1-x}$ As layer from Eq. (7). Using an orthorombic crystal unit cell with 8 atoms and dimensions $\left\{\mathbf{a}_{i}\right\}$ along the $\langle 100\rangle$ directions, we computed the MASAs $f_{\mathrm{Ga}, \mathrm{GaAs}}^{\prime 002}(x, s)$ and $f_{\mathrm{As}, \mathrm{GaAs}}^{\prime 002}(x, s)$ of a strained GaAs unit cell, as well as the MASAs $f_{\text {In,InAs }}^{\prime 002}(x, s)$ and $f_{\mathrm{As}, \text { InAs }}^{\prime 002}(x, s)$ of a strained InAs cell. Note that the same dimensions $\left\{\mathbf{a}_{i}\right\}$ were used for both cells. For the DFT calculation with the WIEN2k program, a sampling of $k$ points with $800 k$ points in the full Brillouin zone was used. The interstitial plane-wave vector cutoff $K_{\max }$ was chosen in such a way that $R_{\mathrm{MT}} K_{\max }=7$. The value of $K_{\max }$ corresponds to an energy cutoff $E_{\max }=10.5$. These values for the number of $k$ points and the interstitial plane wave vector cutoff were found by converging the structure factors of the (002) reflection of GaAs and InAs, respectively. A precision better than $5 \times 10^{-2} \%$ was achieved. The generalized gradient approximation (GGA) of Perdew et al. ${ }^{26}$ was used for the exchange and correlation part of the potential. An acceleration voltage $U=200 \mathrm{kV}$ of the TEM was assumed. The MASAs were calculated for In-concentrations $x$ between 0 and 1 in steps of 0.01 . Strain parameters $s$ of $0,0.5$, and 1 were used. In addition, the calculations were performed for unstrained $\mathrm{In}_{x} \mathrm{Ga}_{1-x} \mathrm{As}$ (i.e., $a_{[100]}=a_{[010]}=a_{[001]}=a_{\text {bulk }}$ ). The computed MASAs have been fitted to the third-order polynomial

$$
f_{\nu, \kappa}^{\prime 002}(x, s)=p_{1}(s) x^{3}+p_{2}(s) x^{2}+p_{3}(s) x+p_{4} .
$$

A list of all polynomial coefficients is given in Table I. Figure 1 shows the composition dependence of the MASAs computed for $s=0.5$. Note that the composition dependence of the MASAs is caused by the composition dependence of the bulk material lattice parameter of $\operatorname{In}_{x} \mathrm{Ga}_{1-x} \mathrm{As}$ and by strain [Eq. (8)]. For comparison, Fig. 1 also contains the atomic scattering amplitudes (ASAs) calculated in the isolated atom approximation. The structure factor of the (002) reflection is according to Eqs. (1), (5), and (6) given by

$$
\begin{array}{rl}
F_{\mathrm{In}_{x} \mathrm{Ga}_{1-\mathrm{A}} \mathrm{As}}^{002} & 4\left\{( 1 - x ) \left(f_{\mathrm{Ga}, \mathrm{GaAs}}^{\prime 002}-{f_{\mathrm{As}, \mathrm{GaAs}}^{\prime 002}}^{002}\right.\right. \\
& \left.+x\left(f_{\mathrm{In}, \mathrm{InAs}}^{\prime 002}-f_{\mathrm{As}, \mathrm{InAs}}^{\prime \prime 002}\right)\right\} .
\end{array}
$$

Note that the MASAs and the structure factor are functions of $x$ and $s$, which is not explicitly indicated in Eq. (17). For simplicity, Debye-Waller temperature factors are not taken into account in Eq. (17).

Figure 2 depicts the resulting composition-dependent 002 structure factor for electron scattering for the different values of the strain parameter $s$. The solid line gives the result we obtain using the bulk material lattice parameter of $\mathrm{In}_{x} \mathrm{Ga}_{1-x}$ As. The zero of $F_{\mathrm{In}_{x} \mathrm{Ga}}^{002} \mathrm{a}_{1-x} \mathrm{As}$ occurs close to $x=0.175$. The difference of the structure factors calculated for $s=0$, 0.5 , and 1 becomes significant for In concentrations larger than approximately 0.3 . Figure 2 also contains the 002 structure factor calculated for $s=0.5$ in the isolated atom approximation with ASAs taken from Ref. 8. Obviously, there is a significant difference between the results of the isolated atom approximation and the DFT computation, which is caused by charge redistribution. Recalling that, according to Eq. (17), the 002 structure factor depends on the difference between the atomic scattering amplitudes of metal and nonmetal atoms, this can be understood from Fig. 1, which shows that the difference between MASAs and ASAs is larger for As than for $\mathrm{Ga}$ and In.

\section{The effect of static atomic displacements}

\section{Theoretical considerations}

Besides charge redistribution, the structure factors of $\mathrm{In}_{x} \mathrm{Ga}_{1-x}$ As are also affected by local structural distortions. Indeed, in any III-V alloy where atoms having different covalent radii share at least one sublattice, there exists an average perfect periodic structure, but the equilibrium positions of the atoms are displaced from its sites [although these static atomic displacements (SDs) are small enough for any atom to be unambiguously assigned to a given site]. Whereas for bulk materials the average structure is cubic sphalerite (with a lattice parameter equal to the average parameter of 
TABLE I. Table of polynomial coefficients for the modified atomic scattering amplitudes of strained $\operatorname{In}_{x} \mathrm{Ga}_{1-x}$ As. The parameter $s$ describes the strain state according to Eq. (8). The bulk material lattice parameter was used for the column "bulk." All values are given in units of (nanometers).

\begin{tabular}{|c|c|c|c|c|c|c|}
\hline Crystal & Element & & $s=0$ & $s=0.5$ & $s=1$ & Bulk \\
\hline \multirow[t]{8}{*}{ GaAs } & \multirow[t]{4}{*}{$\mathrm{Ga}$} & $p_{1}$ & $-7.725 E-4$ & $1.304 E-3$ & $2.933 E-3$ & $2.619 E-4$ \\
\hline & & $p_{2}$ & $1.789 E-3$ & $6.326 E-4$ & $2.738 E-3$ & $-1.009 E-3$ \\
\hline & & $p_{3}$ & $4.295 E-2$ & $5.282 E-2$ & $6.602 E-2$ & $3.205 E-2$ \\
\hline & & $p_{4}$ & $5.384 E-1$ & $5.383 E-1$ & $5.383 E-1$ & $5.383 E-1$ \\
\hline & \multirow[t]{4}{*}{ As } & $p_{1}$ & $-9.497 E-4$ & $7.797 E-4$ & $1.760 E-3$ & $3.178 E-4$ \\
\hline & & $p_{2}$ & $4.945 E-4$ & $-7.600 E-4$ & $8.080 E-4$ & $-1.547 E-3$ \\
\hline & & $p_{3}$ & $4.446 E-2$ & $5.300 E-2$ & $6.426 E-2$ & $3.471 E-2$ \\
\hline & & $p_{4}$ & $5.907 E-1$ & $5.906 E-1$ & $5.907 E-1$ & $5.907 E-1$ \\
\hline \multirow[t]{8}{*}{ InAs } & \multirow[t]{4}{*}{ In } & $p_{1}$ & $-7.940 E-4$ & $1.446 E-3$ & $3.050 E-3$ & $2.278 E-4$ \\
\hline & & $p_{2}$ & $1.271 E-3$ & $1.904 E-4$ & $3.182 E-3$ & $-1.372 E-3$ \\
\hline & & $p_{3}$ & $5.889 E-2$ & $7.218 E-2$ & $9.013 E-2$ & $4.348 E-2$ \\
\hline & & $p_{4}$ & $8.311 E-1$ & $8.311 E-1$ & $8.311 E-1$ & $8.311 E-1$ \\
\hline & \multirow[t]{4}{*}{ As } & $p_{1}$ & $-1.403 E-3$ & $9.827 E-4$ & $2.536 E-3$ & $1.902 E-4$ \\
\hline & & $p_{2}$ & $1.442 E-3$ & $-8.390 E-4$ & $-2.023 E-4$ & $-1.411 E-3$ \\
\hline & & $p_{3}$ & $4.306 E-2$ & $5.147 E-2$ & $6.235 E-2$ & $3.443 E-2$ \\
\hline & & $p_{4}$ & $5.831 E-1$ & $5.831 E-1$ & $5.831 E-1$ & $5.830 E-1$ \\
\hline
\end{tabular}

the alloy), for the thinned epitaxial layers considered here, the sphalerite unit cell is homogeneously strained into an orthorhombic cell with lattice parameters a along the $\langle 100\rangle$ directions [Eqs. (7) and (8) ]. Because In and Ga have covalent radii differing by about $14 \%, \mathrm{In}_{x} \mathrm{Ga}_{1-x}$ As is strongly affected by SDs. Detected by extended x-ray absorption fine structure experiments, ${ }^{27}$ the SDs were subsequently shown to have significant effects in $\mathrm{x}$-ray and electron diffraction, ${ }^{28}$ diffraction contrast TEM ${ }^{29}$ and high resolution TEM. ${ }^{30}$

In an alloy with no long range order, and in particular in the random alloys here considered, the SDs destroy the translation symmetry of the crystal and nonzero Fourier compo-

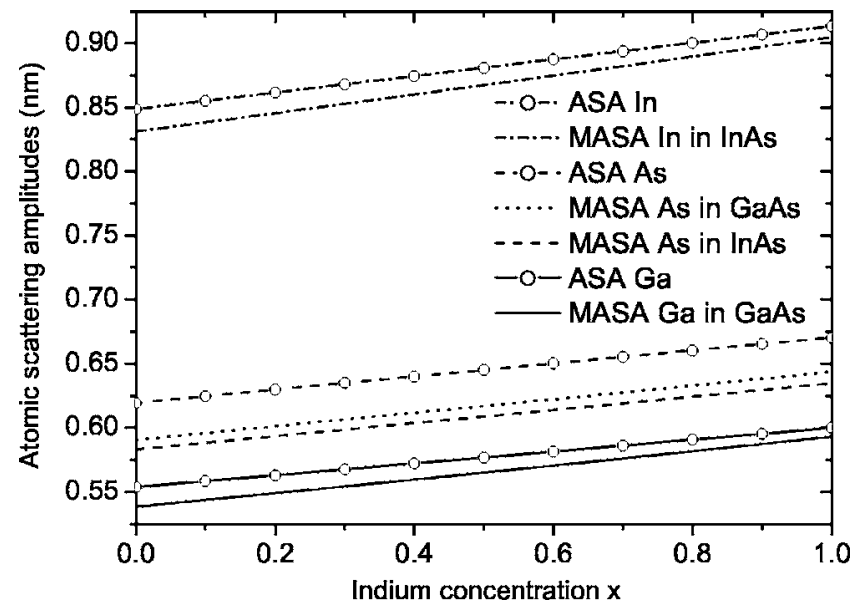

FIG. 1. MASAs $f_{\text {In,InAs }}^{\prime 002}, f_{\mathrm{As}, \mathrm{GaAs}}^{002}, f_{\mathrm{As}, \mathrm{InAs}}^{\prime 002}$, and $f_{\mathrm{Ga}, \mathrm{GaAs}}^{\prime 002}$, as well as corresponding ASAs calculated in the isolated atom approximation from Doyle and Turner (see Ref. 8), plotted vs the Inconcentration $x$. The curves were calculated for a strain factor of $s=0.5$ and an acceleration voltage of the electron beam of $200 \mathrm{kV}$. nents of the potential exist for vectors not belonging to the reciprocal lattice (which, experimentally, gives rise to diffuse scattering). However, there still exists a long range ordered perfect average lattice and an associated reciprocal lattice. In the isolated atom approximation, the average $h k l$ Fourier component of the potential [Eq. (1)] becomes

$$
\begin{aligned}
V^{h k l}(\mathbf{a})= & \frac{h_{\mathrm{P}}^{2}}{2 \pi m_{0} e N \Omega(\mathbf{a})} \sum_{n=1}^{N} \sum_{\nu=1}^{2} \sum_{j=1}^{4} D_{j, \nu, n}^{h k l}(\mathbf{a}) f_{j, \nu, n}^{h k l}(\mathbf{a}) \\
& \times \exp \left[2 \pi i \mathbf{g}^{h k l}(\mathbf{a}) \cdot\left\{\mathbf{r}_{j, \nu}(\mathbf{a})+\mathbf{u}_{j, \nu, n}\right\}\right],
\end{aligned}
$$

where we now also sum over the $N$ different unit cells of a given crystal and take into account that site $j, \nu$ of cell $n$ is

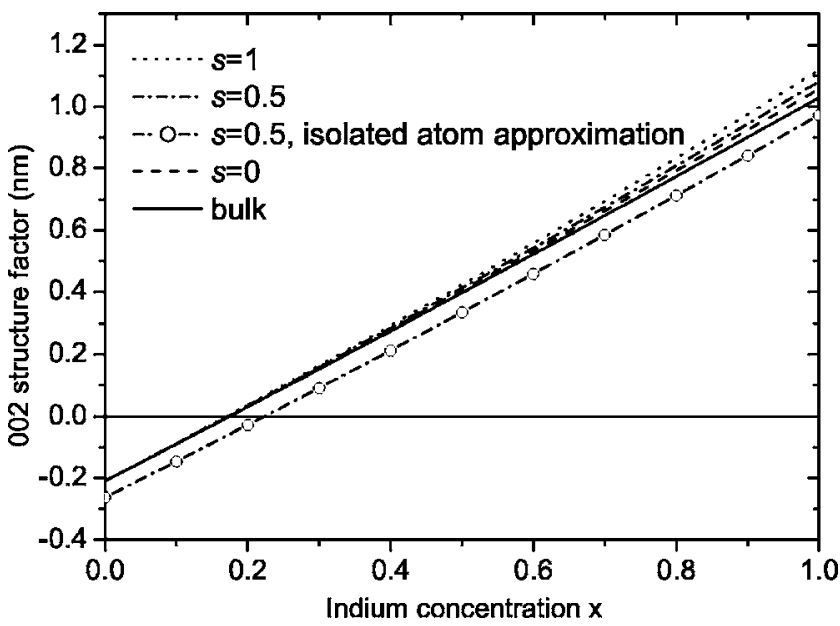

FIG. 2. Structure factor for electron scattering of the 002 reflection in $\operatorname{In}_{x} \mathrm{Ga}_{1-x} \mathrm{As}$, plotted vs the In-concentration $x$ (acceleration voltage $200 \mathrm{kV}$ ). 
occupied by a particular atom (Ga or In on the mixed sublattice, As only on the nonmetal sublattice) displaced from its average site by $\mathbf{u}_{j, \nu, n} \cdot{ }^{31}$ Note that the actual position of perfect lattice site $j, \nu, n$ is the sum of $\mathbf{r}_{j, \nu}$ and of the position vector of the origin of cell $n$ : however, the latter term disappears via its scalar product with reciprocal vector $\mathbf{g}^{h k l}$. Using this approximation, we recently carried out a comprehensive study which showed that the SDs may significantly modify the SFs of the "weak" reflections [such that $h+k+l$ $\equiv 2(\bmod 4)]$ in a large number of III-V and II-VI cubic alloys. ${ }^{13}$

Because both sublattices are distorted and because the SDs do not depend only on the nature of a few nearest neighbors (NNs) but are correlated over distances of several nm, ${ }^{29}$ the calculation of statistically significant SD-related properties cannot rely on oversimplifying assumptions (such as the nondistortion of the mixed sublattice), which would allow to consider small unit cells. Instead, they must be based on averages taken over large simulated crystals. However, for such large supercells, DFT calculations become impractical.

To take both charge redistribution and SDs into account, we thus adopt the following approach. We simulate large $\mathrm{In}_{x} \mathrm{Ga}_{1-x} \mathrm{As}$ supercells with various In compositions $x$ and in various strain states $s$. Atoms are first placed at the sites of a perfect average crystal with parameters $\mathbf{a}(x, s)[\mathrm{Eq}$. (7)]. The overall dimensions of the supercell are taken (and subsequently kept) equal to large multiples (typically 50) of the components of $\mathbf{a}$. The SDs are then calculated numerically by using the extended valence force field (VFF) model, ${ }^{32,33}$ as detailed previously. ${ }^{13,28}$ Finally, we calculate $V^{h k l}(\mathbf{a})$ from Eq. (18) by inserting the SDs $\mathbf{u}_{j, v, n}$ and by replacing the standard atomic scattering amplitudes by quantities derived from the MASAs calculated in Sec. II B 2 for the same values of $x$ and $s$. Namely, for a metal atom $(\nu=1)$, which has four As NNs, we simply replace $f_{j, 1, n}^{h k l}(\mathbf{a})$ by $f_{\text {Ga.GaAs }}^{\prime h k l}(x, s)$ if site $j, 1, n$ is occupied by a $\mathrm{Ga}$ atom and by $f_{\operatorname{In}, \operatorname{InAs}}^{\prime h k l}(x, s)$ if it is occupied by an In atom. For an As atom at site $j, 2, n$ having $p_{j, n}^{\mathrm{M}}$ metal NNs of species $\mathrm{M}$ ( with $\left.p_{j, n}^{\mathrm{Ga}}+p_{j, n}^{\mathrm{In}}=4\right)$, we replace $f_{j, 2, n}^{h k l}(\mathbf{a})$ by $\frac{1}{4}\left[p_{j, n}^{\mathrm{Ga}} f_{\mathrm{As}, \mathrm{GaAs}}^{\prime h k l}(x, s)+p_{j, n}^{l_{\mathrm{n}}} f_{\mathrm{As}, \operatorname{InAs}}^{\prime h k}(x, s)\right]$. Omitting here again Debye-Waller factors and the dependence of $\mathbf{g}^{h k l}$ and $\mathbf{r}_{j, \nu}$ on a, the structure factor is then obtained from Eqs. (5) and (18) as

$$
\begin{aligned}
F^{h k l}= & \frac{1}{N} \sum_{n=1}^{N}\left(\sum_{j=1}^{4}\left[\sigma_{j, n}^{\mathrm{Ga}} f_{\mathrm{Ga}, \mathrm{GaAs}}^{\prime h k l}+\sigma_{j, n}^{\mathrm{In}} f_{\mathrm{In}, \mathrm{InAs}}^{\prime h k l}\right]\right. \\
& \times \exp \left[2 \pi i \mathbf{g}^{h k l} \cdot\left\{\mathbf{r}_{j, 1}+\mathbf{u}_{j, 1, n}\right\}\right] \\
& +\sum_{j=1}^{4}\left[\frac{p_{j, n}^{\mathrm{Ga}}}{4} f_{\mathrm{As}, \mathrm{GaAs}}^{\prime h k l}+\frac{p_{j, n}^{\mathrm{In}}}{4} f_{\mathrm{As}, \mathrm{InAs}}^{\prime h k l}\right] \\
& \left.\times \exp \left[2 \pi i \mathbf{g}^{h k l} \cdot\left\{\mathbf{r}_{j, 2}+\mathbf{u}_{j, 2, n}\right\}\right]\right)
\end{aligned}
$$

where $\sigma_{j, n}^{\mathrm{M}}=1$ if site $j, 1, n$ is occupied by metal atom $\mathrm{M}$, and $\sigma_{j, n}^{\mathrm{M}}=0$ if not. Noting that for any reciprocal lattice vector, $\exp \left(2 \pi i \mathbf{g}^{h k l} \cdot \mathbf{r}_{j, v}\right)=\tau_{v} / 4$ for any $j=1$ to 4 , Eq. (19) rewrites

$$
\begin{aligned}
F^{h k l}= & \tau_{1}\left[(1-x) d_{\mathrm{Ga}, \mathrm{As}}^{h k l} f_{\mathrm{Ga}, \mathrm{GaAs}}^{\prime h k l}+x d_{\mathrm{In}, \mathrm{As}}^{h k l} f_{\mathrm{In}, \mathrm{InAs}}^{\prime h k l}\right]+\tau_{2}[(1 \\
& \left.-x) d_{\mathrm{As}, \mathrm{Ga}}^{h k l} f_{\mathrm{As}, \mathrm{GaAs}}^{\prime h k l}+x d_{\mathrm{As}, \mathrm{In}}^{h k l} f_{\mathrm{As}, \mathrm{InAs}}^{\prime h k l}\right],
\end{aligned}
$$

where the static displacement correction factors are defined as

$$
\begin{aligned}
& d_{\mathrm{M}, \mathrm{As}}^{h k l}=\frac{1}{4 N x_{\mathrm{M}}} \sum_{n=1}^{N} \sum_{j=1}^{4} \sigma_{j, n}^{\mathrm{M}} \exp \left[2 \pi i \mathbf{g}^{h k l} \cdot \mathbf{u}_{j, 1, n}\right] \\
& d_{\mathrm{As}, \mathrm{M}}^{h k l}=\frac{1}{4 N x_{\mathrm{M}}} \sum_{n=1}^{N} \sum_{j=1}^{4} \frac{p_{j, n}^{\mathrm{M}}}{4} \exp \left[2 \pi i \mathbf{g}^{h k l} \cdot \mathbf{u}_{j, 2, n}\right]
\end{aligned}
$$

with $x_{\mathrm{M}}$ the concentration of metal $\mathrm{M}\left(x_{\mathrm{Ga}}=1-x, x_{\mathrm{In}}=x\right)$. At variance with the usual "static Debye-Waller factors," the static displacement correction factors depend not only on the nature of the atoms but also on their environments. In Eq. (21), the number of nonzero terms summed is precisely $4 N x_{\mathrm{M}}$, so that for large enough random crystals, $d_{\mathrm{M}, \mathrm{As}}$ should not depend on the details of the distributions of the atoms; the same holds for $d_{\mathrm{As}, \mathrm{M}}$. However, these quantities depend on $x$ and $s$ because the statistical properties of the SDs depend on these parameters. Note that $\tau_{1} / 4=1$ for any reflection and that $\tau_{2} / 4=-1$ for any weak reflection. In particular, for 002, Eq. (20) rewrites

$$
\begin{aligned}
F^{002}= & 4\left\{(1-x)\left(d_{\mathrm{Ga}, \mathrm{As}}^{002} f_{\mathrm{Ga}, \mathrm{GaAs}}^{\prime 002}-d_{\mathrm{As}, \mathrm{Ga}}^{002} f_{\mathrm{As}, \mathrm{GaAs}}^{\prime 002}\right)\right. \\
& +x\left(d_{\mathrm{In}, \mathrm{As}}^{002} f_{\mathrm{In}, \mathrm{InAs}}^{\prime 002}-d_{\mathrm{As}, \mathrm{In}}^{002} f_{\mathrm{As}, \mathrm{InAs}}^{\prime 002}\right\} .
\end{aligned}
$$

In Eq. (23), the MASAs and the static displacement correction factors, and therefore the structure factor, depend on $x$ and $s$. Finally, each $d_{\mathrm{A}, \mathrm{B}}^{002} f_{\mathrm{A}, \mathrm{B}}^{\prime 002}$ product can be multiplied by the appropriate Debye-Waller factor.

\section{Results}

We simulated 13 alloy crystals containing one million atoms $(N=50)$ with different In concentrations, each in the four strain states mentioned in Sec. II B 2. The SD field was found by minimizing the VFF energy and the static displacement correction factors were then calculated by using Eqs. (21) and (22). Figures 3 and 4 show the variations of these factors for As and metal atoms, respectively.

That the static displacement correction factors are less than one has been discussed in Ref. 13. Moreover, at any concentration $x, d_{\mathrm{As}, \mathrm{M}}^{002}$ differs more from one than $d_{\mathrm{M}, \mathrm{As}}^{002}$. This is because, in such ternary alloys, the homogeneous sublattice (here, the As sublattice) is more distorted than the mixed (metal) sublattice. ${ }^{34}$ The behavior of the correction factors in dilute alloys can be understood as follows. For $x \rightarrow 0$, the alloy is nearly pure GaAs with a cubic unit cell irrespective of its strain state (since the layers considered are epitaxially deposited on GaAs). The only distortions occur around an isolated In atom, which, for sake of symmetry, stays at its average site whereas the four surrounding As atoms (and less so, the more distant atoms) are displaced from theirs. Hence, $d_{\mathrm{In}, \mathrm{As}}^{002} \rightarrow 1$ whereas $d_{\mathrm{As}, \mathrm{In}}^{002} \neq 1$ since all As atoms with In NN are displaced. Most of the III-V pairs are Ga-As so that $d_{\mathrm{Ga}, \mathrm{As}}^{002}$ and $d_{\mathrm{As}, \mathrm{Ga}}^{002} \rightarrow 1$. For $x \rightarrow 1$, the alloy is nearly pure InAs. The only distortions occur around an isolated $\mathrm{Ga}$ atom. 


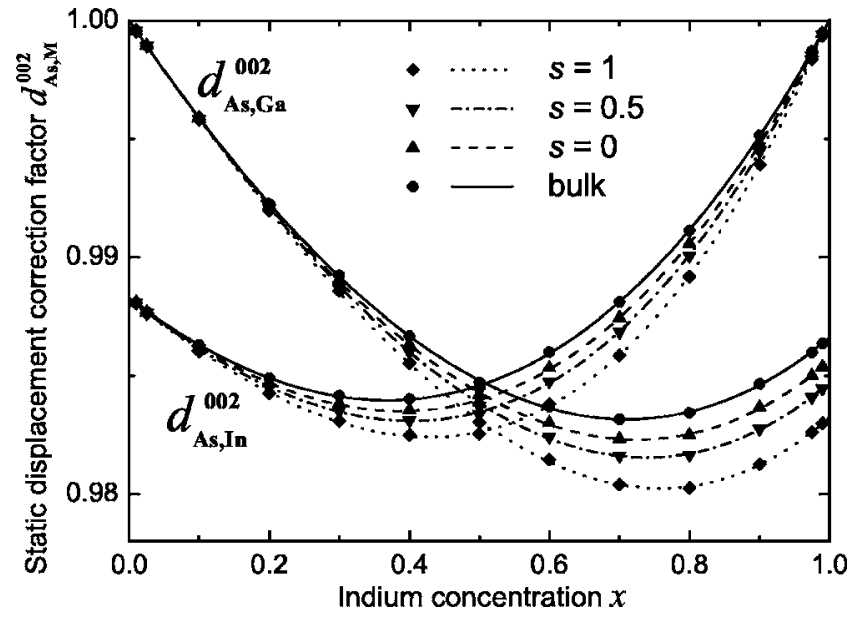

FIG. 3. Static displacement correction factors to the 002 structure factor for As atoms in $\mathrm{In}_{x} \mathrm{Ga}_{1-x} \mathrm{As}$, as a function of the Inconcentration $x$. The lines are polynomial fits to the results of the VFF calculations (symbols).

The preceding discussion applies again to these atoms and to the majority atoms (In and As with In NN), so that $d_{\mathrm{Ga}, \mathrm{As}}^{002}, d_{\mathrm{In}, \mathrm{As}}^{002}$ and $d_{\mathrm{As}, \mathrm{In}}^{002} \rightarrow 1$. The only atoms systematically displaced are the As with a $\mathrm{Ga} N$, so that $d_{\mathrm{As}, \mathrm{Ga}}^{002} \neq 1$. However, since the average tetragonal distortion of the near-InAs crystal deposited on GaAs depends on its strain state $s$, so do the SDs and the limit of $d_{\mathrm{As}, \mathrm{Ga}}^{002}$.

For use in Eq. (23), the correction factors were fitted to a third-order polynomial. Using the same notation as in Eq. (16), the corresponding coefficients are listed in Table II. Figure 5 shows the quantity to be added to the SF calculated according to Eq. (17) (Fig. 2) to obtain the SF taking into account both charge redistribution and static atomic displacements [Eq. (23)]; this difference has the sign opposite to that found in Ref. 13 because of the change of origin in the unit cell. Since it is increased by the SD correction, the SF consequently vanishes at lower In concentrations which, for all strain states considered, are close to $x=0.16$. Note that this

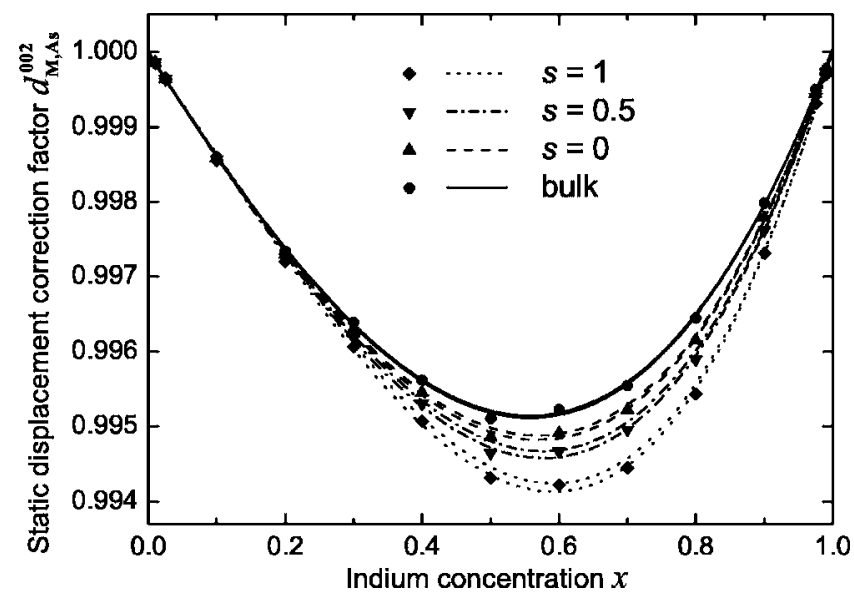

FIG. 4. Same as Fig. 3 for Ga and In atoms. For each strain parameter $s$, the lower curve corresponds to $\mathrm{Ga}$ and the upper one to In. Since the VFF results for Ga and In are very close, only the former are shown. shift is due not to the mere existence of SDs, but to the difference of magnitude between the SDs of As and metal atoms.

\section{DISCUSSION}

Figure 6 shows 002 structure factors computed by different methods as a function of the In concentration, where the respective bulk material lattice parameter is used. The dashed line depicts the structure factor derived using Eq. (17) and the atomic scattering amplitudes from Doyle and Turner. ${ }^{8}$ The structure factor vanishes at an In concentration of $22.5 \%$. The full and dotted lines show the dependence of the 002 structure factor derived from Eq. (23) using the polynomial fit coefficients from Table I for the calculation of the MASAs. For the dotted line, the SDs were neglected by setting the SD correction factors equal to 1 [in this case Eq. (23) simplifies to Eq. (17)], whereas for the full line the polynomial fit coefficients for SD correction factors from Table II were used. The structure factors derived from our computations are clearly shifted with respect to those computed from the Doyle and Turner atomic scattering amplitudes. ${ }^{8}$ The 002 structure factor vanishes at $17.5 \%$ for the computations without SDs. The shift of the structure factor towards larger values is caused by redistribution of electrons due to bonding of atoms in the crystal, which is of course ignored in the computation of the atomic scattering factors of Doyle and Turner, ${ }^{8}$ but is taken into account in our computation of the MASAs. Additionally taking into account the SDs, the structure factor is again shifted slightly to larger values and vanishes at $16.4 \%$.

The derivation of the electron structure factor from Eq. (23) using the MASAs the SD correction factors includes an approximation. The MASAs are computed by taking into account the average unit cell distortion but not the SDs. Therefore, the structure factors might additionally be influenced by a redistribution of charge due to SDs. A better procedure to incorporate SDs and charge redistribution would be to derive the SDs of a large alloy supercell (with typically one million atoms), as described in Sec. II C 2, compute by DFT the x-ray scattering factors of this supercell, including the SDs, and finally calculate the electron structure factors similar to Eq. (12). However, the computational effort required for the DFT calculation is too large for such a supercell.

Such computations can nevertheless be performed for ordered alloys and this allowed us to check the validity of our approach. To this end, we simulated ordered alloys with unit cells consisting of 4 As atoms, $p$ In atoms and $(4-p) \mathrm{Ga}$ atoms, with $p=1,2$, or 3 , and lattice parameter corresponding to the bulk alloy composition, respectively, $x=0.25,0.5$, and 0.75 . In this case, the atoms of the same sublattice (metal or nonmetal) were considered nonequivalent. Consequently, the lapw3 program lists separate contributions to the $\mathrm{x}$-ray scattering factor for all individual atoms: $X_{j, \operatorname{In}}^{h k l}(j=1$ to $p)$ for the $p$ In atoms, $X_{j, \mathrm{Ga}}^{h k l}[j=1$ to $(4-p)]$ for the $(4-p)$ Ga atoms and $X_{j, \mathrm{As}}^{h k l}(j=1$ to 4$)$. To describe this situation, we rewrite Eq. (11) to give the X-ray scattering structure factor $X^{h k l}$ of the ordered-alloy InGaAs crystal as 
TABLE II. Table of polynomial coefficients for the static displacement correction factors $d_{\mathrm{A}, \mathrm{B}}^{002}$ to the 002 SF of strained $\mathrm{In}_{x} \mathrm{Ga}_{1-x} \mathrm{As}$, with A the atom considered and B its nearest neighbor. Parameter $s$ as in Table I.

\begin{tabular}{|c|c|c|c|c|c|c|}
\hline$B$ & $A$ & & $s=0$ & $s=0.5$ & $s=1$ & Bulk \\
\hline \multirow[t]{4}{*}{ As } & \multirow[t]{4}{*}{$\mathrm{Ga}$} & $p_{1}$ & 0.0123 & 0.0144 & 0.0188 & 0.0095 \\
\hline & & $p_{2}$ & 0.0019 & -0.0004 & -0.0057 & 0.0051 \\
\hline & & $p_{3}$ & -0.0142 & -0.0139 & -0.0131 & -0.0145 \\
\hline & & $p_{4}$ & 1.0000 & 1.0000 & 1.0000 & 1.0000 \\
\hline \multirow[t]{4}{*}{$\mathrm{Ga}$} & \multirow[t]{4}{*}{ As } & $p_{1}$ & 0.0104 & 0.0126 & 0.0169 & 0.0078 \\
\hline & & $p_{2}$ & 0.0185 & 0.0151 & 0.0087 & 0.0223 \\
\hline & & $p_{3}$ & -0.0433 & -0.0430 & -0.0423 & -0.0435 \\
\hline & & $p_{4}$ & 1.0000 & 1.0000 & 1.0000 & 1.0000 \\
\hline \multirow[t]{4}{*}{ As } & \multirow[t]{4}{*}{ In } & $p_{1}$ & 0.0120 & 0.0139 & 0.0185 & 0.0093 \\
\hline & & $p_{2}$ & 0.0021 & -0.0001 & -0.0057 & 0.0053 \\
\hline & & $p_{3}$ & -0.0141 & -0.0138 & -0.0128 & -0.0145 \\
\hline & & $p_{4}$ & 1.0000 & 1.0000 & 1.0000 & 1.0000 \\
\hline \multirow[t]{4}{*}{ In } & \multirow[t]{4}{*}{ As } & $p_{1}$ & 0.0123 & 0.0157 & 0.0224 & 0.0086 \\
\hline & & $p_{2}$ & 0.0221 & 0.0191 & 0.0124 & 0.0254 \\
\hline & & $p_{3}$ & -0.0227 & -0.0230 & -0.0230 & -0.0222 \\
\hline & & $p_{4}$ & 0.9883 & 0.9883 & 0.9882 & 0.9883 \\
\hline
\end{tabular}

$$
X^{h k l}=\sum_{j=1}^{p} X_{j, \mathrm{In}}^{h k l}+\sum_{j=1}^{4-p} X_{j, \mathrm{Ga}}^{h k l}+\sum_{j=1}^{4} X_{j, \mathrm{As}}^{h k l}+X_{\mathrm{Out}}^{h k l} .
$$

The structure factor $F_{\mathrm{InGaAs}}^{h k l}$ for electron scattering is

$$
\begin{aligned}
F_{\text {InGaAs }}^{h k l}= & \frac{e^{2} m_{0}\left(1+\frac{e U}{m_{0} c^{2}}\right)}{2 \pi h_{\mathrm{P}}^{2} \epsilon_{0}\left(\mathbf{g}^{h k l}\right)^{2}} \\
& \times\left\{\sum_{\nu=1}^{2} \sum_{j=1}^{4} Z_{j, \nu} \exp \left[2 \pi i \mathbf{g}^{h k l} \cdot\left(\mathbf{r}_{j, \nu}+\mathbf{u}_{j, \nu}\right)\right]-X^{h k l}\right\},
\end{aligned}
$$

where the static atomic displacement $\mathbf{u}_{j, \nu}$ of atom $j$ in sub-

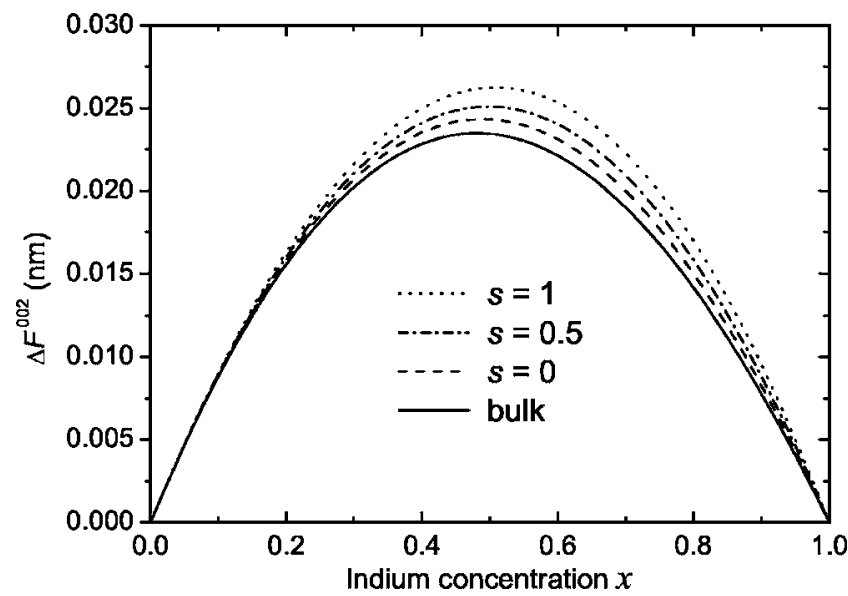

FIG. 5. Differences of the 002 structure factors for strained $\mathrm{In}_{x} \mathrm{Ga}_{1-x} \mathrm{As}$ calculated with and without taking into account the static atomic displacements, as a function of the In concentration. lattice $\nu$ is either set to zero for a calculation without SDs or computed using the VFF model (the usual periodic boundary conditions ensure the formation of an ordered alloy). This approach, which in the following will be called the "direct DFT" approach, circumvents the two approximations made in Eqs. (6) and (23), because the unit cell used for the DFT

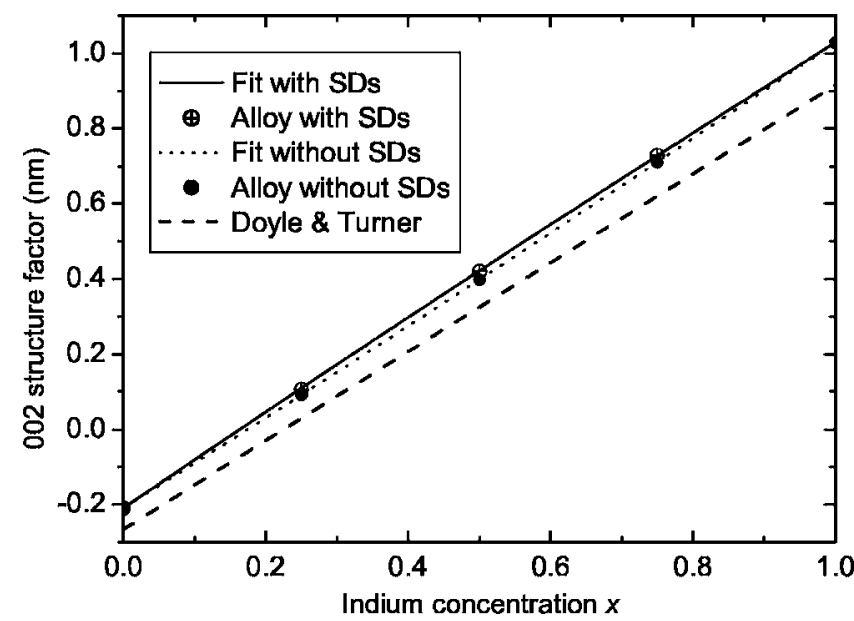

FIG. 6. 002 structure factors plotted as a function of the In concentration. The dashed line was computed using the atomic scattering amplitudes of Doyle and Turner (see Ref. 8). The dotted and full lines were computed from Eqs. (16) and (23) using the polynomial fit coefficients in Table I for the calculation of the MASAs. For the full line the SD correction factors were calculated with the polynomial fit coefficients in Table II, whereas for the dotted line the SDs were not taken into account. The filled and open circles indicate structure factors that were directly obtained by DFT computations of ordered alloys using Eq. (25) with (open circles with crosses) and without (filled circles) taking into account SDs. 
TABLE III. Structure factors (in nanometers) for the three 002 type reflections, calculated for three $\mathrm{In}_{x} \mathrm{Ga}_{1-x}$ As bulk ordered alloys, without ("no SD") and with ("with SD") static atomic displacements. "MASA" values were obtained from Eq. (16) and Table I (no SD) and corrected according to Eq. (23) by using static displacements factors calculated specifically for the ordered alloys (with SD). "DFT" values correspond to the direct DFT approach which uses Eq. (25).

\begin{tabular}{cccccc}
\hline \hline$x$ & Reflection & $\begin{array}{c}\text { No SD } \\
\text { MASA }\end{array}$ & $\begin{array}{c}\text { No SD } \\
\text { DFT }\end{array}$ & $\begin{array}{c}\text { With SD } \\
\text { MASA }\end{array}$ & $\begin{array}{c}\text { With SD } \\
\text { DFT }\end{array}$ \\
\hline 0.25 & $200,020,002$ & 0.0913 & 0.0911 & 0.1060 & 0.1060 \\
0.5 & 200,002 & 0.3981 & 0.3977 & 0.3981 & 0.3966 \\
0.5 & 020 & 0.3981 & 0.3977 & 0.4657 & 0.4685 \\
0.75 & $200,020,002$ & 0.7107 & 0.7101 & 0.7297 & 0.7294 \\
\hline \hline
\end{tabular}

calculation now contains a mixture of In and Ga atoms together with the static displacements of all the atoms. The "direct DFT approach" thus allows a check of the validity of the two approximations by comparing its results with those obtained with MASAs via Eqs. (6) and (23).

In Table III, we compare the 002-type structure factors calculated either by using the tabulated MASAs or with the direct DFT approach, for crystals without and with SDs. For the calculations from MASAs, we used Eqs. (16) and (23) with SD correction factors calculated specifically from the optimized cells of the ordered alloys, since the SD fields of ordered and disordered alloys are different: the metal atoms remain undisplaced in the three ordered crystals whereas the As atoms are displaced along $\langle 111\rangle$ directions for $x=0.25$ or 0.75 , but only along 020 in the particular crystal chosen for $x=0.5$. We thus expect all 002-type structure factors to be equal for $x=0.25$ or 0.75 , whereas for $x=0.5$ only the 020 factor should be affected by the SDs. Table III shows that this is indeed the case. Moreover, there is an excellent agreement between the direct DFT calculations and the calculations from MASAs. This fully justifies our method of taking into account separately the charge redistribution (through the MASAs) and the SDs (through the correction factors). In addition, we compare in Fig. 6 the structure factors calculated for disordered alloys (from MASAs) and ordered alloys (direct DFT approach). These agree again very well whether the SDs are ignored or not, provided, however, that, in the latter case and for $x=0.5$, the value for the disordered alloy (where the three 200-type directions are equivalent) is compared with the DFT value averaged over the three 002-type directions (see Table III).

We also checked the SDs calculated in the VFF model by a comparison with an $a b$ initio optimization. For that purpose, we used one of the cells of the ordered alloys with SDs and tried to optimize the structure with the "mini" program of the WIEN2k software package. We found that no geometry optimization was necessary, indicating that the forces on the atoms are small and that the SDs are in a good agreement with $a b$ initio structure optimization. The forces on the atoms in the ordered alloy with SDs were smaller than $4 \times 10^{-11} \mathrm{~N}$, i.e., two orders of magnitude smaller than for the cell without SDs.

Computations within the DFT formalism are always dependent upon approximations for the exchange and correlation part of the potential, because the exact form of the ex- change and correlation functional is unknown. To estimate the error on the structure factors due to these approximations, we computed the structure factors of GaAs and InAs using the local density approximation (LDA) in the parametrization of Ceperly and Alder ${ }^{35}$ instead of the GGA. We found that the structure factor for GaAs differs by only $0.20 \%$ from the GGA value, whereas for InAs the computations resulted in a deviation of $0.17 \%$.

In principle, our value for GaAs can also be compared with experimental determinations. We are aware of two measurements of the 002 structure factor for electrons, made by convergent beam electron diffraction. ${ }^{11,36}$ Both experiments actually yield the Fourier component $V_{\mathrm{GaAs}}^{002}$ of the scattering potential for an electron accelerating voltage $U=120 \mathrm{kV}$. We convert these values to structure factors via Eq. (5) and to $U=200 \mathrm{kV}$. We also divide the value of Ref. 36, which pertains to $T=100 \mathrm{~K}$, by the appropriate Debye-Waller factor. ${ }^{37}$ This yields $F_{\mathrm{GaAs}}^{002}=-0.183 \pm 0.004 \mathrm{~nm}$ and $-0.184 \mathrm{~nm}$, respectively, which differs by about $12 \%$ from our calculated value for GaAs. However, the difficulties inherent in extracting the structure factors from the experiments ${ }^{38}$ should be borne in mind when making such comparisons. Moreover, no measurement for alloys seems to have been made so far.

Using the DFT theory and SDs computed with the extended VFF model, we found that the structure factor of $\mathrm{In}_{x} \mathrm{Ga}_{1-x} \mathrm{As}$ vanishes at an In concentration $x=0.164$. Cagnon et $a .^{5}$ and Patriarche et al. ${ }^{6}$ measured values of $x=0.17$ and $x=0.18$, respectively. However, these experiments correspond to an interpolated minimum rather than to an observed zero of the 002 diffracted intensity and no estimation of the experimental errors is quoted. Furthermore, we expect a small shift of our theoretical value if Debye-Waller factors are used. Namely, since the Debye-Waller factor for In is larger than the Debye-Waller factors for $\mathrm{Ga}$ and $\mathrm{As}$, the In concentration causing the structure factor to vanish should be shifted to higher values. Using calculated Debye-Waller factors, ${ }^{37}$ we estimate this shift to about $\delta x=0.002$ in In concentration at $T=300 \mathrm{~K}$, which brings our results very close to the experimental determination of Cagnon et al. ${ }^{5}$

\section{SUMMARY}

In summary, we showed that the 002 structure factor is strongly influenced by redistribution of electrons due to bonding of atoms in a crystal and by local structural distor- 
tions. We demonstrated how the redistribution of electrons can be taken into account by using newly defined quantities, the MASAs, instead of the usual atomic scattering amplitudes, and how the SDs can be incorporated via SD correction factors. The dependences of the MASAs and of the SD correction factors upon composition were fitted to third-order polynomials, and fitting coefficients were provided. In agreement with experimental measurements, the 002 structure fac- tor computed using MASAs and SD correction factors vanishes for an In concentration of $16.4 \%$.

\section{ACKNOWLEDGMENT}

A.R. and D.L. acknowledge financial support from the FWO-Vlaanderen under Contract No. G.0425.05.
*Electronic address: rosenauer@ifp.uni-bremen.de

${ }^{1}$ J. Verbeeck and S. Van-Aert, Ultramicroscopy 101, 207 (2004).

${ }^{2}$ R. Bierwolf, M. Hohenstein, F. Phillipp, O. Brandt, G. E. Crook, and K. Ploog, Ultramicroscopy 49, 273 (1993).

${ }^{3}$ A. Rosenauer, S. Kaiser, T. Reisinger, J. Zweck, W. Gebhardt, and D. Gerthsen, Optik (Stuttgart) 102, 63 (1996).

${ }^{4}$ P. M. Petroff, J. Vac. Sci. Technol. 14, 973 (1974).

${ }^{5}$ J. Cagnon, P. A. Buffat, P. A. Stadelmann, and K. Leifer, Inst. Phys. Conf. Ser. 180, 203 (2003).

${ }^{6}$ G. Patriarche, L. Largeau, J.-C. Harmand, and D. Gollub, Appl. Phys. Lett. 84, 203 (2004).

${ }^{7}$ A. Rosenauer, Transmission Electron Microscopy of Semiconductor Nanostructures-An Analysis of Composition and Strain, Springer Tracts in Modern Physics 182 (Springer, Heidelberg, 2003).

${ }^{8}$ P. A. Doyle and P. S. Turner, Acta Crystallogr. A24, 390 (1968).

${ }^{9}$ A. Weickenmeier and H. Kohl, Acta Crystallogr. A47, 590 (1991)

${ }^{10}$ Z. Su and P. Coppens, Acta Crystallogr. A53, 749 (1997).

${ }^{11}$ J. M. Zuo, J. C. H. Spence, and M. O’Keeffe, Phys. Rev. Lett. 61, 353 (1988).

${ }^{12}$ F. Glas, Inst. Phys. Conf. Ser. 180, 191 (2003).

${ }^{13}$ F. Glas, Philos. Mag. 84, 2055 (2004).

${ }^{14}$ A. J. F. Metherell, Electron Microscopy in Materials Science (Commission of European Communtities, Luxemburg, 1975).

${ }^{15}$ C. J. Humphreys, Rep. Prog. Phys. 42, 1825 (1979).

${ }^{16}$ J. M. Cowley and A. F. Moodie, Acta Crystallogr. 10, 609 (1957).

${ }^{17}$ J. M. Cowley and A. F. Moodie, Acta Crystallogr. 12, 353 (1959).

${ }^{18}$ J. Howie and M. J. Whelan, Proc. R. Soc. London, Ser. A 263, 217 (1961).

${ }^{19}$ J. C. H. Spence and J. M. Zuo, Electron Microdiffraction (Plenum Press, New York, 1992).
${ }^{20}$ International Tables for Crystallography, edited by T. Hahn (Kluwer Academic, Dordrecht, 1991), Vol. B

${ }^{21}$ J. Cowley, Electron Diffraction Techniques (Oxford University Press, Oxford, 1992).

${ }^{22}$ C. Humphreys and E. Bithell, Electron Diffraction Techniques (Oxford University Press, Oxford, 1992).

${ }^{23}$ J. Stahn, M. Möhle, and U. Pietsch, Acta Crystallogr. B54, 231 (1998).

${ }^{24}$ P. Blaha, K. Schwarz, G. K. H. Madsen, D. Kvasnicka, and J. Luitz, WIEN2k, An Augmented Plane Wave + Local Orbitals Program for Calculating Crystal Properties (Karlheinz Schwarz, Techn. Universität Wien, Austria, 2001), ISBN 3-9501031-1-2 (2001).

${ }^{25}$ N. F. Mott, Proc. R. Soc. London, Ser. A A127, 658 (1930).

${ }^{26}$ J. P. Perdew, K. Burke, and M. Ernzerhof, Phys. Rev. Lett. 77, 3865 (1996).

${ }^{27}$ J. C. Mikkelsen, Jr. and J. B. Boyce, Phys. Rev. B 28, 7130 (1983).

${ }^{28}$ F. Glas, C. Gors, and P. Hénoc, Philos. Mag. B 62, 373 (1990).

${ }^{29}$ F. Glas, Phys. Rev. B 51, 825 (1995).

${ }^{30}$ F. Glas, Ultramicroscopy 60, 91 (1995).

${ }^{31}$ J. M. Cowley, Diffraction Physics (North-Holland, Amsterdam, 1975).

${ }^{32}$ P. N. Keating, Phys. Rev. 145, 637 (1966).

${ }^{33}$ R. M. Martin, Phys. Rev. B 1, 4005 (1970).

${ }^{34}$ M. Podgórny, M. T. Czyżyk, A. Balzarotti, P. Letardi, N. Motta, A. Kisiel, and M. Zimnal-Starnawska, Solid State Commun. 55, 413 (1985).

${ }^{35}$ D. M. Ceperley and B. J. Alder, Phys. Rev. Lett. 45, 566 (1980).

${ }^{36}$ J. Pacaud and J. M. Zuo, Proceedings of the 13th European Microscopy Congress, Antwerp 2004 (Belgian Society for Microscopy, Liège, 2004).

${ }^{37}$ H. X. Gao and L.-M. Peng, Acta Crystallogr. A55, 926 (1999).

${ }^{38}$ J. E. Bernard and A. Zunger, Phys. Rev. Lett. 62, 2328 (1989). 